\title{
Reduced Neurite Density in the Brain and Cervical Spinal Cord in Relapsing-Remitting Multiple Sclerosis: a NODDI Study.
}

\author{
S. Collorone ${ }^{1}$, N. Cawley ${ }^{1}$, F. Grussu ${ }^{1,2}$, F. Prados ${ }^{1,2}$, F. Tona ${ }^{1}$, A. Calvi ${ }^{1,3}$, B. \\ Kanber $^{2}$, T. Schneider ${ }^{1,4}$, L. Kipp ${ }^{1,5}$, H. Zhang², D. C. Alexander ${ }^{2}$, A.J. \\ Thompson ${ }^{1}$, A. Toosy ${ }^{1}$, C.A.M. Gandini Wheeler-Kingshott ${ }^{1,6,7}$, O. Ciccarelli ${ }^{1,8}$
}

${ }^{1}$ NMR Research Unit, Queen Square MS Centre, Department of

Neuroinflammation, University College London Institute of Neurology, Faculty of

Brain Sciences, UCL, London, United Kingdom

${ }^{2}$ Centre for Medical Image Computing (CMIC), Department of Computer

Science, University College London, London, UK

${ }^{3}$ Department of Pathophysiology and Transplantation, Neurodegenerative

Disease Unit, La Fondazione IRCCS Ospedale Maggiore Policlinico Mangiagalli

e Regina Elena, University of Milan, Milan, Italy

${ }^{4}$ Philips UK, Guildford, Surrey, UK

${ }^{5}$ Stanford MS Center, Department of Neurology and Neurological Sciences,

Stanford University, Palo Alto, California, USA 
${ }^{6}$ Department of Brain and Behavioural Sciences, University of Pavia, Pavia, Italy

${ }^{7}$ Brain MRI 3T Research Centre, IRCCS Mondino Foundation, Pavia, Italy

${ }^{8}$ National Institute for Health Research, University College London Hospitals, Biomedical Research Centre, London, UK

Corresponding author:

Dr Sara Collorone

Queen Square Multiple Sclerosis Centre

Department of Neuroinflammation, UCL Institute of Neurology

1st Floor, Russell Square House, 10-12 Russell Square

London

WC1B 5EH

e-mail: s.collorone@ucl.ac.uk

Twitter handle:@SaraCollorone

Tel.: +44 (0) 7400254777 


\section{KEYWORDS}

Multiple sclerosis; relapsing/remitting; quantitative MRI; NODDI; diffusionweighted MRI; axonal loss. 


\section{ABSTRACT}

Background: Multiple sclerosis (MS) affects both brain and spinal cord. However, studies of the neuraxis with advanced MRI are rare because of long acquisition times. We investigated neurodegeneration in MS brain and cervical spinal cord using neurite orientation dispersion and density imaging (NODDI). Objective: to investigate possible alterations, and their clinical relevance, in neurite morphology along the brain and cervical spinal cord of relapsingremitting MS patients.

Methods: 28 relapsing-remitting MS patients and 20 healthy controls underwent brain and spinal cord NODDI at 3T. Physical and cognitive disability was assessed. Individual maps of orientation dispersion index (ODI) and neurite density index (NDI) in brain and spinal cord were obtained. We examined differences in NODDI measures between groups and the relationships between NODDI metrics and clinical scores using linear regression models adjusted for age, sex and brain tissue volumes or cord cross-sectional area.

Results: Patients showed lower NDI in the brain normal-appearing white matter and spinal cord white matter than healthy controls. In patients, a lower NDI in the spinal cord white matter was associated with higher disability. 
Conclusions: Reduced neurite density occurs in the neuraxis but, especially when affecting the spinal cord, it may represent a mechanism of disability in MS.

\section{INTRODUCTION}

Neurodegeneration represents a major component of multiple sclerosis (MS) pathology and it can affect the whole neuraxis ${ }^{1}$, brain and spinal cord, from the relapsing-remitting stage of $\mathrm{MS}^{2}$. Quantitative magnetic resonance imaging (MRI) biomarkers of the brain ${ }^{3}$ and spinal cord 4 may reflect neurodegeneration in vivo and, thus, may be used as outcome measures in clinical trials targeting neuroprotection in $\mathrm{MS}^{5}$. However, it is unclear whether brain or spinal cord pathological alterations are the main correlates of disability in $\mathrm{MS}^{6}$. To address 
this question, studies of the neuraxis with quantitative MRI should be carried out, but these are rare because of the long acquisition times. Neurite orientation dispersion and density imaging (NODDI) is a multicompartmental model of diffusion $\mathrm{MRI}^{7}$ that is more specific than DTI in capturing the microstructural substrates possibly underpinning neurodegeneration ${ }^{8}$. Specifically, NODDI provides two indices of neurite morphology, the neurite density index (NDI) and the neurite orientation dispersion index (ODI). NDI estimates the fraction of axons and dendrites within the neural tissue: it is primarily sensitive to axonal loss, and therefore neurodegeneration, with other changes, such as demyelination, also contributing to its value ${ }^{9}$. ODI quantifies the variability of neurite orientations: a high orientation dispersion occurs when neurites are dispersed in space; therefore, it becomes meaningless when measured in voxels corresponding to cerebrospinal fluid (CSF).

A few studies have applied NODDI to the brain of MS patients. They all reported a decrease in the NDI in both the white matter (WM) lesions ${ }^{10-12}$ and normalappearing $\mathrm{WM}^{10-13}$ in MS patients compared with healthy controls (HCs). ODI was also found to be altered, predominantly in WM lesions, but in different directions depending on the study ${ }^{10-12}$. Alterations in NODDI indices were 
correlated with physical disability, as measured by the Expanded Disability Status Scale (EDSS) $)^{11,13}$.

However, no study has investigated correlations between NODDI alterations and cognitive impairment, ${ }^{14}$ which is associated with neurodegeneration, ${ }^{15}$ and the impact of WM lesions on the observed NODDI abnormalities is unknown. Additionally, a previous study ${ }^{11}$ used a unique 3T scanner with gradient strength up to $300 \mathrm{mT} / \mathrm{m}$, a technology not widely available in the clinical setting, whose results are difficult to compare and/or to replicate.

Another key question is whether NODDI can be used to investigate in vivo neurodegeneration in the spinal cord of MS patients. We have previously demonstrated the feasibility of NODDI application to the healthy spinal cord ${ }^{16}$. A preliminary observation in a small number of patients $(\mathrm{N}=6)^{17}$ has suggested abnormal ODI and NDI metrics in the spinal cord of MS patients when compared with controls.

Thus, a characterization of the neurite density and orientation dispersion in the whole neuraxis of MS patients is lacking, although this would contribute to understanding if the brain and spinal cord share common pathological abnormalities. Therefore, we applied NODDI to the brain and cervical cord of 
relapsing-remitting MS (RRMS) patients and HCs, using a clinical 3T scanner. To determine if the underlying abnormalities in the neurite morphology, as captured by NODDI, contribute to disability, we assessed the correlations between NODDI indices and physical and cognitive disability.

\section{MATERIALS AND METHODS}

\section{Patients' characteristics and clinical assessments}

We enrolled 28 consecutive patients who fulfilled the following inclusion criteria: diagnosis of RRMS, age between 18 and 65 years, absence of relapses or corticosteroid treatment in the three months before imaging and no other known medical condition affecting the central nervous system (CNS). Twenty age- and sex-matched HCs were also recruited.

Patients were assessed with the EDSS. All subjects underwent the MS functional composite (MSFC) score, which includes the 9-Hole Peg Test, the Timed 25-Foot Walk test and the Paced Auditory Serial Addition Test, as well as the brief cognitive assessment for MS (BICAMS) ${ }^{18}$ including Symbol Digit Modalities Test (SDMT), California Verbal Learning Test-II (CVLT-II) and Brief Visuospatial Memory Test-Revised (BVMT-R). Anxiety and depression were tested as possible confounders with the Hospital Anxiety and Depression Scale $(\operatorname{HADS})^{19}$. 
For the MSFC total score, z-scores were calculated according to the National Multiple Sclerosis Society Task Force guidelines ${ }^{20}$.

Written informed consent, approved by the local research ethics committee, was obtained for all participants.

\section{MRI protocol}

Brain and spinal cord MRI scans were performed in the same session at 3T using a Philips Achieva MRI system (Philips Healthcare, Best, the Netherlands) with RF multi-transmit technology, Quasar Dual gradient set of $40 / 80 \mathrm{mT} / \mathrm{m}$ and with a 32-channel coil for brain acquisition and a 16-channel neurovascular receive-only radiofrequency coil for the spinal cord (Table 1). The spinal cord protocol was acquired before the brain protocol in all subjects.

Brain NODDI cardiac-gated diffusion-weighted images were acquired using a spin-echo echo-planar imaging sequence. The diffusion protocol consisted of 3 b-shells and gradient directions isotropically distributed: $b$-value $=300 \mathrm{~s} / \mathrm{mm}^{2}$ and 8 directions; $b$ value $=1000 \mathrm{~s} / \mathrm{mm}^{2}$ and 30 directions; $b$ value $=2855 \mathrm{~s} / \mathrm{mm}^{2}$ and 60 directions. Eight interleaved non-diffusion-weighted $(b=0)$ images were also acquired.

The cervical cord was imaged in the axial-oblique plane (i.e., slices perpendicular to the longitudinal axis of the cord) with the center of the imaging volume positioned at the level of C2-3 intervertebral disc. Moreover, to reduce 
motion artifacts during scanning and improve image quality, an MR compatible cervical collar was worn by all subjects ${ }^{21}$.

For NODDI, the acquisition protocol for all scans relied on a reduced field-ofview (FOV), cardiac gated PGSE ZOOM-EPI sequence, acquiring two diffusionweighted high angular resolution imaging (HARDI) ${ }^{22}$ b-shells of thirty measurements at $b=1000 \mathrm{~s} / \mathrm{mm}^{2}$ and sixty measurements at $b=2855 \mathrm{~s} / \mathrm{mm}^{2}$ and 3 interleaved $b=0$ measurement, respectively ${ }^{16}$. A total of 20 slices were acquired with a total acquisition time of approximately $35 \mathrm{~min}$. Simulations, not included in this paper, revealed that $3 b=0$ measurements suffice to estimate the correct $b=0$ offset level $95 \%$ of times, with a precision of $7.8 \%$, against precision of $4.9 \%$, with $8 b=0$ measurements, with realistic spinal cord SNR levels ${ }^{16,23}$.

\section{Conventional MRI post-processing}

T2 hyperintense lesions were outlined in each subject using the semiautomated edge finding tool from JIM v6.0 (Xinapse systems, Aldwincle, UK) and the lesion volume $(\mathrm{ml})$ was computed. The lesion masks were co-registered to the 3D-T1 images ${ }^{24}$ and then the 3D-T1 images were filled using a non-local patch match lesion filling technique ${ }^{25}$. For brain extraction, tissue segmentation and parcellation, we used Geodesic Information Flows (GIF) ${ }^{26}$. 
The upper cervical cord cross-sectional area (CSA) was measured from the 3DFFE scans of the cervical cord: an active surface model was applied using JIM v6.0 to obtain the CSA by averaging the area of three contiguous $5 \mathrm{~mm}$ axial slices, centered on $\mathrm{C} 2 / 3$ disc $^{27,28}$. Spinal cord lesions were identified as delineated areas of hyperintensity on the 3D-FFE sequence and counted.

\section{Diffusion-weighted data processing}

\section{Brain}

Each diffusion-weighted image was corrected for eddy current-induced distortions and subject movements using eddy in FSL6.0(FMRIB, Oxford, UK) ${ }^{29}$. For the NODDI fitting, we used the Matlab (The MathWorks, Inc., Natick, Massachusetts, USA) NODDI toolbox (http://nitrc.org/projects/noddi toolbox) with the default settings to generate ODI and NDI.

The segmented tissue masks and lesion masks were then registered to the NODDI maps to extract the tissue-specific NODDI values.

Spinal cord

Diffusion-weighted data were pre-processed and corrected for motion ${ }^{16}$. For each subject, the whole cord was segmented on the mean $b=0$ volume by using a semi-automatic active surface method ${ }^{27}$ implemented in JIM V6.0. The fitting cord mask was then eroded slice-by-slice and cropped to the 6 central slices creating the whole-cord mask. Subsequently, the spinal cord grey matter (GM) 
and WM, as well as dorsal, lateral and ventral WM sub-regions masks, were manually outlined in each subject on the average DW image which was obtained by averaging the diffusion-weighted images acquired for gradient directions at angle less or equal to 50 degrees with respect to the longitudinal axis of the spinal cord, according to the procedure described by Kearney and colleagues $^{30}$ (Supplemental Material).

The NODDI model was fitted within the whole cord mask (before slice wise erosion) by using the NODDI MATLAB toolbox. NODDI indices were then calculated within the manually segmented spinal cord tissue masks. Voxels with isotropic volume fraction (i.e. CSF voxels) $>0.99$ were excluded from the analysis.

All the brain and spinal cord NODDI images underwent quality checks and were excluded based on motion artifacts, noticeable signal drifts on the $b=0$ signal. Examples of the brain and spinal cord NODDI maps are given in Figure 1.

\section{Statistical analysis}

Group differences in demographic characteristics were assessed using the twosample t-test for continuous variables and the chi-square test for categorical variables.

We applied a linear regression model to investigate the differences between 
patients and HCs in brain tissue volumes (i.e., WM and GM tissue volumes), CSAs, clinical tests and NODDI metrics, adjusting for age and sex. When looking for differences in NODDI metrics, the models were repeated including specific brain tissue volumes (when brain comparisons were tested) or CSA (when spinal cord comparisons) to account for the possible influence of atrophy. In patients, we used linear regression models to investigate associations between NODDI metrics and brain volumes and spinal cord CSA, and disability (i.e., MSFC and BICAMS), adjusting for age and sex. Only the NODDI metrics and the clinical tests that showed significant differences between patients and HCs were entered in these models. In case of significant associations between NODDI metrics and disability scores, the regressions were re-run adjusting for the brain parenchymal fraction and the spinal cord CSA.

Since the EDSS score and lesion loads (brain lesion volume and spinal cord lesion count) are not normally distributed, we used the Spearman's rho correlation coefficient for the associations between NODDI metrics and these parameters.

Stata v. 14.1 (Stata Corporation, College Station, Texas, USA) was used. Results associated with a $p<0.05$ were considered statistically significant and reported. Due to the exploratory nature of the study, the statistical inference of multiple comparisons was not performed. 


\section{RESULTS}

Out of 28 patients and $20 \mathrm{HCs}$ who underwent the MRI protocol (Table 2), we included into the analysis the brain MRI data of 24 patients and $16 \mathrm{HCs}$ and the spinal cord data of 27 patients and $18 \mathrm{HCs}$ (the remainder scans were discarded after quality checks and/or for incomplete MRI protocol). In comparison to HCs, RRMS patients showed brain and spinal cord atrophy (Table 2) as well as lower scores on the BICAMS, and MSFC (Table 3). The BICAMS and PASAT scores did not correlate with the HADS scores.

Patients showed lower NDI in the brain normal-appearing WM than HCs $(B=-$ $0.021,95 \%$ confidence interval (Cls): -0.041 to $-0.002, p=0.032$ ). In the spinal cord, patients showed lower NDI in the whole WM $(B=-0.07,95 \%$ Cls:- 0.11 to $0.03, p=0.001$ ), particularly in the dorsal and lateral WM sub-regions, and a lower ODI in the dorsal WM $(B=-0.04,95 \% C l s:-0.07$ to -0.1$)$ than $\mathrm{HCs}$ (Figure 2). These differences remained significant after adjusting for WM brain tissue volume and CSA, respectively (Table 4). NDI was lower in the spinal cord GM of RRMS patients than $\mathrm{HCs}(\mathrm{B}=-0.04,95 \% \mathrm{Cls}:-0.07$ to $-0.006, \mathrm{p}=0.021)$, but this difference became not significant when adjusting for CSA. 
In patients, a lower NDI in the whole WM and dorsal sub-region of the spinal cord correlated with higher EDSS score $\left(r_{s=}=0.46 p=0.015\right.$ and $r_{s=}=0.48, p=0.012$, respectively) (Figure 3), while there was a weak correlation between lower ODI in the dorsal column and higher EDSS $\left(r_{s=}-0.39 p=0.045\right)$.

In patients, lower NDI in the brain normal-appearing WM was associated with a higher brain lesion load $\left(r_{s=}=0.52, p=0.009\right)($ Table 5-6).

\section{DISCUSSION}

Using a clinical scanner, we found evidence of neurite integrity loss, as captured by NDI, in both the spinal cord and the brain of patients with MS when compared to HCs.

In the cervical cord of MS patients, a reduced density of nerve fibers (NDI) was detected in the whole cord, especially in the lateral and dorsal WM columns. We also found a lower dispersion of neurites (ODI) in the dorsal columns of patients when compared to controls, as opposed to the finding of a different research group, which studied only six MS patients ${ }^{17}$. A low ODI value may be related to a reduction in the angular variations of axons, possibly caused by demyelination, as well as to the contribution of WM lesions, not excluded in our 
analysis, that have shown low ODI values in previous studies from our group ${ }^{10,31}$ and are often present in the dorsal columns of MS patients ${ }^{32}$. The results of the brain NODDI comparisons between groups mirrored the spinal cord findings, with a lower NDI in the brain normal-appearing WM of patients when compared to HCs. In the brain, we found a relationship between lower NDI and higher lesion load, thus extending the findings of previous studies ${ }^{11,13}$. As alterations in the normal-appearing WM of MS patients can be characterized by diffuse axonal injury ${ }^{33}$, our results may support the hypothesis that this axonal damage is due to the degeneration of axons transected in focal lesions $s^{34}$.

The most interesting result of our study is that lower NDI in the spinal cord WM was associated with greater disability, as measured by EDSS. This suggests that a reduced neurite density, which is a known component of neurodegeneration, may contribute to neurological impairment in MS. When reflecting on the absence of a significant correlation between low neurite density and increased disability in the brain, it is important to consider that long axons are highly compacted in the WM columns of the spinal cord, where they mediate neurological functions. Therefore, the alterations in the axon 
microstructure detected by NODDI in the spinal cord WM are more likely to correlate with the EDSS, which is heavily weighted towards walking ability, than the global reduced NDI observed in the brain normal-appearing WM. This interpretation is supported by a previous study carried out in MS which reported that the EDSS was related to reduced NDI only in specific brain WM regions, such as the corpus callosum and internal capsule, where long-tract axons are found ${ }^{13}$.

Finally, for the first time, we investigated possible correlations between cognitive performance and NODDI metrics. However, measures of cognition did not correlate with abnormal NODDI parameters. Since patterns of orientation abnormalities detected by diffusion imaging in the cortex seem to be related to cognitive functions ${ }^{35}$, further studies analyzing NODDI in specific cortical regions and linking them with the corresponding cognitive domains are needed.

\section{Limitations}

A possible limitation of our analysis is that we could not exclude MS lesions from the spinal cord NODDI maps; therefore, it is expected that NDI from the lesional tissue contributes to the observed reduced NDI. However, the relationship between spinal cord NDI and disability was independent of spinal 
cord lesion load, suggesting that NODDI may reflect clinically meaningful alterations in the WM independently of visible lesions.

Secondly, the length of the protocol does not allow the use of NODDI in routine clinical practice. However, changes in scanner hardware and acquisition protocols may lead to faster acquisition time, with the possibility of translating this technique from research centres to the clinical setting.

Finally, in the spinal cord, our relatively coarse resolution may have led to high levels of within-voxel partial volume effects, and the presence of highly anisotropic voxels may increase partial volume along the superior-inferior direction, thereby leading to higher values of ODI, as compared to brain WM areas with similar fibre organization.

Nonetheless, previous works ${ }^{7,12}$ have demonstrated that the echo times used in this study suffices to obtain an image quality suitable for group comparisons at the relatively low resolution employed here. Future work aiming at clinical translation is warranted to improve signal-to-noise and hence achieve a better resolution and mitigate partial volume effects.

In conclusion, the neurite integrity loss, which is a crucial element of neurodegeneration, occurs in both the brain and spinal cord WM in MS, but mainly when affecting the cervical cord, it may contribute to physical disability. 
This raises the potential of using spinal cord NDI metric as a marker of disability in MS so that it can be used to assess the efficacy of neuroprotective treatments and to provide insights into the mechanisms of disability in MS. 


\section{ACKNOWLEDGEMENTS}

Authors acknowledge the researchers at the National Institute for Health Research University College London Hospitals Biomedical Research Centre who supported this project.

\section{FUNDING AND COMPETING INTERESTS}

This study was funded by EPSRC (EP/I027084/1, EP/M020533/1, EP/N018702/1), ISRT (IMG006) and UK MS Society (984).

SC reports grant from Rosetrees Trust (MS632) and she was awarded a MAGNIMS-ECTRIMS fellowship in 2016. FG reports grant from the European Union's Horizon 2020 research and innovation programme under grant agreement No. 634541 and from the Engineering and Physical Sciences Research Council (EP/M020533/1 and EP/R006032/1). FP is a non-clinical Guarantors of the Brain fellow and has also received honoraria from Bioclinica Inc. AC was awarded a MAGNIMS-ECTRIMS fellowship in 2018. TS is an employee of Philips UK. DCA reports from the European Union's Horizon 2020 research and innovation programme under grant agreement Nos 666992 and 634541, as well as EPSRC grants G007748, 1027084, M020533, N018702. AJT has received honoraria/support for travel for consultancy from Eisai, Biogen (Optum Insight), Hoffman La Roche, MedDay, TEVA, Almirall, and Excemed. He received support for travel for consultancy as chair of the International 
Progressive MS Alliance Scientific Steering Committee, and member of the National MS Society (USA) Research Programs Advisory Committee. He receives an honorarium from SAGE Publishers as Editor-in-Chief of Multiple Sclerosis Journal and a free subscription from Elsevier as a board member for the Lancet Neurology. Support from the NIHR UCLH Biomedical Research Centre is acknowledged. ATT has received speaker honoraria from Biomedia, Sereno Symposia International Foundation, Bayer and meeting expenses from Biogen Idec and is the UK PI for two clinical trials sponsored by MEDDAY pharmaceutical company (MD1003 in optic neuropathy [MS-ON and progressive MS [MS-SPI2]). CAMGW-K has received research funding from EPSRC, UK MS Society, ISRT, Wings for Life and Craig H. Neilsen Foundation for the INSPIRED study, the European Union's Horizon 2020 research and innovation programme under grant agreement No 634541 . OC is a member of the Scientific Advisory Boards of Teva, Novartis, Roche, Biogen and Merck; she has received funding for travel or speaker honoraria from Teva, Novartis, Roche, Biogen and Merck; she is Associate Editor of Neurology and she serves on the Editorial Board of Multiple Sclerosis Journal; she receives research support from the NIHR UCLH/UCL Biomedical Research Centre, NIHR, MS Society of Great Britain and Northern Ireland, National MS Society, Rosetrees 
Trust. MPW serves as a consultant for Roche, Novartis, and Biogen. NC, FT, $\mathrm{BK}, \mathrm{LK}$ and $\mathrm{HZ}$ have nothing to disclose.

\section{REFERENCES}


1. Kutzelnigg A, Lassmann $\mathrm{H}$. Pathology of multiple sclerosis and related inflammatory demyelinating diseases. Handb Clin Neurol 2014; 122: 1558.

2. Lassmann H. Pathogenic Mechanisms Associated With Different Clinical Courses of Multiple Sclerosis. Front Immunol 2019; 9: 3116.

3. Cortese R, Collorone S, Ciccarelli O, et al. Advances in brain imaging in multiple sclerosis. Ther Adv Neurol Disord 2019; 12: 175628641985972.

4. Moccia M, Ruggieri S, lanniello A, et al. Advances in spinal cord imaging in multiple sclerosis. Ther Adv Neurol Disord 2019; 12: 175628641984059 .

5. Tur C, Moccia M, Barkhof F, et al. Assessing treatment outcomes in multiple sclerosis trials and in the clinical setting. Nat Rev Neurol 2018; 14: 75-93.

6. Cohen AB, Neema M, Arora A, et al. The relationships among MRIdefined spinal cord involvement, brain involvement, and disability in multiple sclerosis. J Neuroimaging 2012; 22: 122-8.

7. Zhang $\mathrm{H}$, Schneider $\mathrm{T}$, Wheeler-Kingshott $\mathrm{CA}$, et al. NODDI: Practical in vivo neurite orientation dispersion and density imaging of the human brain. Neuroimage 2012; 61: 1000-1016.

8. Timmers I, Roebroeck A, Bastiani M, et al. Assessing Microstructural 
Substrates of White Matter Abnormalities: A Comparative Study Using DTI and NODDI. PLoS One 2016; 11: e0167884.

9. Jespersen SN, Kroenke CD, Østergaard L, et al. Modeling dendrite density from magnetic resonance diffusion measurements. Neuroimage 2007; 34: 1473-86.

10. Schneider T, Brownlee WJ, Zhang H, et al. Sensitivity of multi-shell NODDI to multiple sclerosis white matter changes: a pilot study. Funct Neurol 2017; 32: 97.

11. Granberg $\mathrm{T}$, Fan $\mathrm{Q}$, Treaba CA, et al. In vivo characterization of cortical and white matter neuroaxonal pathology in early multiple sclerosis. Brain 2017; 140: 2912-2926.

12. De Santis S, Bastiani M, Droby A, et al. Characterizing Microstructural Tissue Properties in Multiple Sclerosis with Diffusion MRI at $7 \mathrm{~T}$ and $3 \mathrm{~T}$ : The Impact of the Experimental Design. Neuroscience 2019; 403: 17-26.

13. Spanò B, Giulietti G, Pisani V, et al. Disruption of neurite morphology parallels MS progression. Neurol - Neuroimmunol Neuroinflammation 2018; 5: e502.

14. Chiaravalloti ND, DeLuca J. Cognitive impairment in multiple sclerosis. Lancet Neurol 2008; 7: 1139-51.

15. Zivadinov R, Sepcic J, Nasuelli D, et al. A longitudinal study of brain 
atrophy and cognitive disturbances in the early phase of relapsingremitting multiple sclerosis. J Neurol Neurosurg Psychiatry 2001; 70: $773-80$.

16. Grussu F, Schneider T, Zhang H, et al. Neurite orientation dispersion and density imaging of the healthy cervical spinal cord in vivo. Neuroimage 2015; 111: 590-601.

17. By S, Xu J, Box BA, et al. Application and evaluation of NODDI in the cervical spinal cord of multiple sclerosis patients. Neurolmage Clin 2017; 15: 333-342.

18. Langdon DW, Amato MP, Boringa J, et al. Recommendations for a brief international cognitive assessment for multiple sclerosis (BICAMS). Mult Scler J 2012; 18: 891-898.

19. Pais Ribeiro JL, Martins da Silva A, Vilhena E, et al. The hospital anxiety and depression scale, in patients with multiple sclerosis. Neuropsychiatr Dis Treat 2018; Volume 14: 3193-3197.

20. Fischer JS, Rudick RA, Cutter GR, et al. The Multiple Sclerosis Functional Composite measure (MSFC): an integrated approach to MS clinical outcome assessment. Mult Scler J 1999; 5: 244-250.

21. Yiannakas MC, Kearney H, Samson RS, et al. Feasibility of grey matter and white matter segmentation of the upper cervical cord in vivo: A pilot 
study with application to magnetisation transfer measurements.

Neuroimage 2012; 63: 1054-1059.

22. Tuch DS, Reese TG, Wiegell MR, et al. High angular resolution diffusion imaging reveals intravoxel white matter fiber heterogeneity. Magn Reson Med 2002; 48: 577-82.

23. Grussu F, lanuş A, Tur C, et al. Relevance of time-dependence for clinically viable diffusion imaging of the spinal cord. Magn Reson Med 2019; 81: 1247-1264.

24. Hickman SI, Barker GJ, Molyneux PD, et al. Technical note: the comparison of hypointense lesions from 'pseudo-T1' and T1-weighted images in secondary progressive multiple sclerosis. Mult Scler 2002; 8: $433-5$.

25. Prados F, Cardoso MJ, MacManus D, et al. A modality-agnostic patchbased technique for lesion filling in multiple sclerosis. Med Image Comput Comput Assist Interv 2014; 17: 781-8.

26. Cardoso MJ, Modat M, Wolz R, et al. Geodesic Information Flows:

Spatially-Variant Graphs and Their Application to Segmentation and Fusion. IEEE Trans Med Imaging 2015; 34: 1976-1988.

27. Horsfield MA, Sala S, Neema M, et al. Rapid semi-automatic segmentation of the spinal cord from magnetic resonance images: 
application in multiple sclerosis. Neuroimage 2010; 50: 446-55.

28. Cawley N, Tur C, Prados F, et al. Spinal cord atrophy as a primary outcome measure in phase II trials of progressive multiple sclerosis. Mult Scler J 2018; 24: 932-941.

29. Andersson JLR, Sotiropoulos SN. An integrated approach to correction for off-resonance effects and subject movement in diffusion MR imaging. Neuroimage 2016; 125: 1063-1078.

30. Kearney H, Schneider T, Yiannakas MC, et al. Spinal cord grey matter abnormalities are associated with secondary progression and physical disability in multiple sclerosis. J Neurol Neurosurg Psychiatry 2015; 86: $608-614$.

31. Grussu F, Schneider T, Tur C, et al. Neurite dispersion: a new marker of multiple sclerosis spinal cord pathology? Ann Clin Trans/ Neurol 2017; 4: $663-679$.

32. Bag AK, Patel BN, Osman S, et al. Clinico-Radiologic Profile of Spinal Cord Multiple Sclerosis in Adults. Neuroradiol J 2011; 24: 511-518.

33. Lassmann H. Multiple Sclerosis Pathology. Cold Spring Harb Perspect Med 2018; 8: a028936.

34. Dziedzic T, Metz I, Dallenga T, et al. Wallerian Degeneration: A Major Component of Early Axonal Pathology in Multiple Sclerosis. Brain Pathol 
2010; 20: no-no.

35. Muhlert N, Sethi V, Schneider T, et al. Diffusion MRI-based cortical complexity alterations associated with executive function in multiple sclerosis. J Magn Reson Imaging 2013; 38: 54-63. 
Table 1. MRI protocol

\begin{tabular}{|c|c|c|c|c|c|}
\hline & $\begin{array}{l}\text { FOV } \\
\left(\mathrm{mm}^{2}\right)\end{array}$ & $\begin{array}{l}\text { Voxel size } \\
\left(\mathrm{mm}^{3}\right)\end{array}$ & $\begin{array}{l}\text { TR } \\
\text { (ms) }\end{array}$ & $\begin{array}{l}\text { TE } \\
\text { (ms) }\end{array}$ & $\begin{array}{l}\text { Inversion } \\
\text { time (ms) }\end{array}$ \\
\hline \multicolumn{6}{|l|}{ BRAIN } \\
\hline 3D-T1 & $256 \times 256$ & $1.0 \times 1.0 \times 1.0$ & 6.9 & 3.1 & 836.46 \\
\hline PD/T2 & $240 \times 180$ & $1.0 \times 1.0 \times 3.0$ & 4900 & $15 / 85$ & \\
\hline DWla & $192 \times 222$ & $2.5 \times 2.5 \times 2.5$ & 12000 & 91 & \\
\hline \multicolumn{6}{|l|}{ SPINE } \\
\hline 3D-FFE & $240 \times 240 \times 50^{b}$ & $0.5 \times 0.5 \times 5.0$ & 23 & 5 & \\
\hline DWI' & $64 \times 64$ & $1.0 \times 1.0 \times 5.0$ & 12000 & 65.50 & \\
\hline
\end{tabular}

a b-values $300 / 1000 / 2855 \mathrm{~s} / \mathrm{mm}^{2}$ with 6/30/60 isotropically distributed gradient directions; TR is approximate being the sequence cardiac gated (TR=12 heart beats)

${ }^{\mathrm{b}} \mathrm{mm}^{3}$

${ }^{c} \mathrm{~b}$-values $1000 / 2855 \mathrm{~s} / \mathrm{mm}^{2}$. TR is approximate being the sequence cardiac gated (TR=12 heart beats).

Abbreviations: DWI: diffusion-weighted image; FFE: fast field echo; FOV: fieldof-view; TR: repetition time; TE: echo time 
Table 2: Demographic and clinical characteristics of relapsing-remitting multiple sclerosis patients and healthy controls.

\begin{tabular}{|c|c|c|c|}
\hline & $\begin{array}{l}\text { RRMS patients } \\
(\mathrm{N}=28)\end{array}$ & $\begin{array}{l}\text { Healthy } \\
\text { controls } \\
(\mathrm{N}=20)\end{array}$ & p-values \\
\hline Age (years) & $39.4 \pm 6.6$ & $36.6 \pm 12.5$ & 0.21 \\
\hline $\begin{array}{l}\text { Gender } \\
\text { (female:male; \% } \\
\text { female) }\end{array}$ & $23: 5(82)$ & $13: 7(65)$ & 0.17 \\
\hline $\begin{array}{l}\text { Disease duration } \\
\text { (years) }\end{array}$ & $8 \pm 5.6$ & - & - \\
\hline DMDs (N, \%) & $15(54)$ & - & - \\
\hline $\begin{array}{l}\text { Brain GM volume } \\
\text { (ml) }\end{array}$ & $624.7 \pm 41.1$ & $705.6 \pm 80.8$ & $0.001^{a}$ \\
\hline $\begin{array}{l}\text { Brain WM } \\
\text { volume (ml) }\end{array}$ & $422 \pm 33.9$ & $482.7 \pm 80.7$ & $0.012^{a}$ \\
\hline BPF & $0.7 \pm 0.02$ & $0.8 \pm 0.01$ & $<0.0001^{a}$ \\
\hline
\end{tabular}




\begin{tabular}{|l|l|l|l|}
\hline $\begin{array}{l}\text { Spinal cord CSA } \\
\left(\mathrm{mm}^{2}\right)\end{array}$ & $75.8 \pm 6.7$ & $83.6 \pm 8.7$ & $0^{-009^{\mathrm{a}}}$ \\
\hline $\begin{array}{l}\text { Brain WM T2 } \\
\text { lesion volume } \\
(\mathrm{ml}) \text { (median, } \\
\text { range) }\end{array}$ & $11.3(0.3-41.3)$ & - & \\
\hline $\begin{array}{l}\text { WM spinal cord } \\
\text { lesion count } \\
\text { (median, range) }\end{array}$ & $2(0-8)$ & & \\
\hline
\end{tabular}

a linear regression model correcting for age and gender.

Note. Data are means \pm standard deviation, unless otherwise indicated.

Abbreviations: RRMS: relapsing-remitting multiple sclerosis; DMDs: disease modifying drugs; GM: grey matter; BPF brain parenchymal fraction; CSA: crosssectional area; WM: white matter 
Table 3: Disability scales and cognitive tests in relapsing-remitting multiple sclerosis patients and healthy controls.

\begin{tabular}{|l|l|l|l|}
\hline & $\begin{array}{l}\text { RRMS patients } \\
\mathbf{( N = 2 8 )}\end{array}$ & $\begin{array}{l}\text { Healthy controls } \\
(\mathbf{N = 2 0})\end{array}$ & $\mathbf{p}^{\text {-value }}{ }^{\mathbf{a}}$ \\
\hline $\begin{array}{l}\text { EDSS (median, } \\
\text { range) }\end{array}$ & $2.5(1-6.5)$ & & \\
\hline $\begin{array}{l}\text { HADS Anxiety } \\
\text { (median, range) }\end{array}$ & $4(0-21)$ & & \\
\hline $\begin{array}{l}\text { HADS } \\
\text { Depression } \\
\text { (median, range) }\end{array}$ & $3(0-10)$ & $18.4 \pm 5.1$ & $\mathbf{0 . 0 0 6}$ \\
\hline $\begin{array}{l}\text { Mean 9-HPT } \\
\text { (sec.) }\end{array}$ & $23 \pm 5.3$ & $3.9 \pm 0.5$ & $\mathbf{0 . 0 3}$ \\
\hline T25FW (sec.) & $5.8 \pm 3.1$ & $48.6 \pm 9.4$ & 0.053 \\
\hline PASAT & $38.8 \pm 16$ & $0.42 \pm 0.4$ & $\mathbf{0 . 0 1 6}$ \\
\hline MSFC (z-score $)$ & $0.03 \pm 0.6$ & $62.13 \pm 10.2$ & $\mathbf{0 . 0 2 7}$ \\
\hline SDMT & $54.56 \pm 11.2$ & $28.29 \pm 39$ & $\mathbf{0 . 0 0 2}$ \\
\hline BVMT-R & $22.28 \pm 7.5$ & $63.27 \pm 8.2$ & $\mathbf{0 . 0 1 6}$ \\
\hline CVLT-II & $57.80 \pm 11.3$ & & \\
\hline
\end{tabular}

${ }^{a} \mathrm{~A}$ linear regression model was used adjusting for age and sex. 
b Z-scores were computed according to the National MS Society's Clinical Outcomes Assessment Task Force indications using as a reference population our healthy control cohort.

Note. Data are means \pm standard deviation, unless otherwise indicated.

Abbreviations: RRMS: Relapsing-Remitting Multiple Sclerosis; 9-HPT: 9-hole Peg Test; T25FW: timed 25-foot walk; PASAT: Paced Auditory Serial Addition Test; MSFC: Multiple Sclerosis Functional Composite; SDMT: Symbol Digit Modality Test; BVMT-R: Brief Visuospatial Memory Test-Revised; CVLT-II Californian Verbal Learning Test II. 
Table 4: NODDI measures in relapsing-remitting multiple sclerosis patients and healthy controls.

\begin{tabular}{|c|c|c|c|c|}
\hline $\begin{array}{l}\text { NODDI } \\
\text { metric }\end{array}$ & $\begin{array}{l}\text { RRMS } \\
\text { patients }\end{array}$ & $\begin{array}{l}\text { Healthy } \\
\text { controls }\end{array}$ & $\begin{array}{l}\text { Standardize } \\
\text { d B } \\
\text { coefficient } \\
\text { (ß) }\end{array}$ & P-value ${ }^{a}$ \\
\hline \multicolumn{5}{|l|}{ Brain } \\
\hline $\mathrm{N}$ of subjects & 24 & 16 & & \\
\hline NDI WM & $0.57 \pm 0.03$ & $0.60 \pm 0.03$ & -0.35 & 0.032 \\
\hline ODI WM & $0.27 \pm 0.02$ & $0.27 \pm 0.02$ & 0.02 & 0.60 \\
\hline NDI GM & $0.44 \pm 0.02$ & $0.44 \pm 0.01$ & -0.25 & 0.24 \\
\hline ODI GM & $0.47 \pm 0.01$ & $0.47 \pm 0.01$ & 0.22 & 0.73 \\
\hline \multicolumn{5}{|l|}{ Spinal cord } \\
\hline $\mathrm{N}$ of subjects & 27 & 18 & & \\
\hline $\begin{array}{l}\text { NDI Whole } \\
\text { WM }\end{array}$ & $0.52 \pm 0.07$ & $0.58 \pm 0.06$ & -0.45 & 0.015 \\
\hline $\begin{array}{l}\text { NDI Lateral } \\
\text { WM }\end{array}$ & $0.53 \pm 0.07$ & $0.58 \pm 0.06$ & -0.38 & 0.021 \\
\hline
\end{tabular}




\begin{tabular}{|l|l|l|l|l|}
\hline $\begin{array}{l}\text { (N voxels in } \\
\text { the mask }\end{array}$ & & & & \\
$134 \pm 47)$ & & & & \\
WDI Dorsal & $0.52 \pm 0.09$ & $0.59 \pm 0.06$ & -0.38 & 0.020 \\
(N voxels in & & & \\
the mask & & & \\
$106 \pm 28)$ & & & & \\
\hline $\begin{array}{l}\text { NDI Ventral } \\
\text { WM }\end{array}$ & $0.48 \pm 0.09$ & $0.55 \pm 0.07$ & -0.27 & 0.095 \\
$(\mathrm{~N}$ voxels in & & & & \\
the mask & & & & \\
$47 \pm 19)$ & & & & \\
\hline $\begin{array}{l}\text { ODI Whole } \\
\text { WM }\end{array}$ & $0.16 \pm 0.05$ & $0.19 \pm 0.04$ & -0.28 & \\
\hline $\begin{array}{l}\text { ODI Lateral } \\
\text { WM }\end{array}$ & $0.16 \pm 0.06$ & $0.18 \pm 0.04$ & -0.15 & \\
\hline $\mathrm{N}$ voxels in & & & & \\
\hline
\end{tabular}




\begin{tabular}{|l|l|l|l|l|}
\hline ODI Dorsal & $0.14 \pm 0.05$ & $0.18 \pm 0.04$ & -0.39 & 0.024 \\
WM & $\mathrm{N}$ voxels in \\
the mask & & & & \\
$106 \pm 28)$ & & & & \\
\hline $\begin{array}{l}\text { ODI Ventral } \\
\text { WM }\end{array}$ & $0.19 \pm 0.07$ & $0.21 \pm 0.05$ & -0.21 & 0.23 \\
$(\mathrm{~N}$ voxels in \\
the mask \\
$47 \pm 19)$
\end{tabular}

a $A$ linear regression model was used adjusting for age, sex and specific tissue volumes (brain) or cervical cord cross-sectional area (spinal cord). 
Note. Data are means \pm standard deviations. Abbreviations: RRMS: relapsingremitting multiple sclerosis; NDI: neurite dispersion index; ODI: orientation dispersion index; WM: white matter; GM: grey matter. 
Table 5. Associations of NODDI metrics with clinical outcomes (a) and Expanded Disability Status Scale (b)

a)

\begin{tabular}{|l|l|l|l|}
\hline $\begin{array}{l}\text { Dependent } \\
\text { variable }\end{array}$ & $\begin{array}{l}\text { Independent } \\
\text { variable }\end{array}$ & $\begin{array}{l}\text { Standardized B } \\
\text { coefficient }\end{array}$ & p-value \\
\hline BRAIN & NDI WM & -0.09 & 0.56 \\
\hline MSFC & NDI WM & -0.12 & 0.51 \\
\hline CVLT-II & NDI WM & -0.1 & 0.56 \\
\hline BVMT-R & NDI WM & 0.04 & 0.82 \\
\hline SDMT & $\begin{array}{l}\text { Brain lesion } \\
\text { volume }\end{array}$ & -0.42 & 0.06 \\
\hline MSFC & $\begin{array}{l}\text { Brain lesion } \\
\text { volume }\end{array}$ & -0.1 & 0.6 \\
\hline CVLT-II & $\begin{array}{l}\text { Brain lesion } \\
\text { volume }\end{array}$ & -0.09 & 0.68 \\
\hline BVMT-R & $\begin{array}{l}\text { Brain lesion } \\
\text { volume }\end{array}$ & -0.17 & 0.4 \\
\hline SDMT & BPF & 0.53 & 0.034 \\
\hline MSFC & BPF & 0.28 & 0.49 \\
\hline CVLT-II & BPF & 0.33 & 0.28 \\
\hline BVMT-R & BPF & 0.28 & 0.25 \\
\hline SDMT & NDI Whole WM & 0.26 & 0.25 \\
\hline SPINAL CORD & NDI Lateral WM & 0.17 & 0.46 \\
\hline MSFC & NDI Dorsal WM & 0.23 & 0.30 \\
\hline MSFC & NDI Dorsal WM & 0.23 & 0.27 \\
\hline MSFC & CSA & 0.20 & 0.39 \\
\hline MSFC & & \multicolumn{2}{l|}{} \\
\hline MSFC & & \\
\hline
\end{tabular}

b)

\begin{tabular}{|l|l|l|l|}
\hline $\begin{array}{l}\text { Dependent } \\
\text { variable }\end{array}$ & $\begin{array}{l}\text { Independent } \\
\text { variable }\end{array}$ & $\mathbf{r}_{\mathrm{s}}$ coefficient & p-value \\
\hline BRAIN & NDI WM & -0.14 & 0.51 \\
\hline EDSS & \multicolumn{2}{|l|}{} \\
\hline SPINAL CORD
\end{tabular}




\begin{tabular}{|l|l|l|l|}
\hline EDSS & NDI Whole WM & -0.46 & $\mathbf{0 . 0 1 5}$ \\
\hline EDSS & NDI Lateral WM & -0.30 & 0.13 \\
\hline EDSS & NDI Dorsal WM & -0.48 & $\mathbf{0 . 0 1 2}$ \\
\hline EDSS & ODI Dorsal WM & -0.39 & $\mathbf{0 . 0 4 5}$ \\
\hline EDSS & CSA & -0.19 & 0.4 \\
\hline EDSS & SC lesion count & 0.48 & $\mathbf{0 . 0 1 8}$ \\
\hline MSFC & SC lesion count & -0.27 & 0.17 \\
\hline
\end{tabular}

a) Results are from linear regression models adjusted for age and sex. Abbreviations: MSFC: Multiple Sclerosis Functional Composite; CVLT-II: California Verbal Learning Test-II; BVMT-R: Brief Visuospatial Memory TestRevised; SDMT: Symbol Digit Modality Test; NDI: Neurite Density Index; WM: White Matter; BPF: brain parenchymal fraction; CSA: cervical cord crosssectional area; SC: spinal cord.

b) Results are from Spearman's correlation test. As EDSS was correlated with the NDI in the spinal cord, associations between EDSS and measure of spinal cord atrophy and lesion load were assessed.

Abbreviations: EDSS: Expanded Disability Status Scale; NDI: Neurite Density Index; WM: White Matter. 
Table 6. Associations between NODDI metrics in the brain and spinal cord and associations with atrophy measures (a) and lesion load (b)

a)

\begin{tabular}{|l|l|l|l|}
\hline $\begin{array}{l}\text { Dependent } \\
\text { variable }\end{array}$ & $\begin{array}{l}\text { Independent } \\
\text { variable }\end{array}$ & $\begin{array}{l}\text { Standardized B } \\
\text { coefficient }\end{array}$ & p-value \\
\hline NDI WM Brain & BPF & 0.32 & 0.26 \\
\hline NDI WM Spine & CSA & 0.30 & 0.19 \\
\hline NDI WM Brain & NDI WM Spine & 0.04 & 0.84 \\
\hline
\end{tabular}

b)

\begin{tabular}{|l|l|l|l|}
\hline $\begin{array}{l}\text { Dependent } \\
\text { variable }\end{array}$ & $\begin{array}{l}\text { Independent } \\
\text { variable }\end{array}$ & $\mathbf{r}_{\mathbf{s}}$ coefficient & $\mathbf{p}$-value \\
\hline NDI WM Brain & $\begin{array}{l}\text { Brain lesion } \\
\text { volume }\end{array}$ & -0.52 & $\mathbf{0 . 0 0 9}$ \\
\hline NDI WM Spine & SC lesion count & -0.33 & 0.10 \\
\hline
\end{tabular}

a) Results are from linear regression models adjusted for age and gender. Abbreviations: NDI: Neurite Density Index; WM: White Matter; BPF: brain parenchymal fraction; CSA: cervical cord cross-sectional area.

b) Results are from Spearman's correlation test.

Abbreviations: NDI: Neurite Density Index; WM: White Matter; SC: spinal cord. 
Figure 1. Example of NODDI maps

A) Example of brain NODDI maps from one healthy control (top row) and two patients: patient 1 (age 51, EDSS 3) and patient 2 (age 42, EDSS 3.5).

The first column shows the corresponding T2 weighed images and the lesion masks drawn for the two patients. The right panel shows the NODDI metrics: neurite density index (NDI) and orientation dispersion index (ODI). Visually, there is a reduction in NDI in MS lesions compared to the normal-appearing white matter in the two patients, and there are evident reduced NDI in the normal-appearing white matter of the two patients when compared to the healthy control.

B) Example of spine NODDI maps obtained from the same subjects. The first column shows the non-diffusion weighted image derived from the DWI. The right panel shows the NODDI metrics. Visually, there is a reduction in NDI in the white matter of the two patients when compared to the healthy control.

Figure 2. Boxplots of the NODDI metrics showing differences between patients and healthy controls.

Top: Neurite density index (NDI) values in the brain normal-appearing white matter of multiple sclerosis patients compared to healthy controls.

${ }^{*}$ p-value $<0.05$ 
Bottom: Neurite density index (NDI) values in the spinal cord white matter of multiple sclerosis patients compared to healthy controls. ${ }^{*} p$-values $<0.05$ Abbreviations: NDI: neurite density index; WM: white matter; MS: multiple sclerosis

Figure 3. Relationship between the EDSS and the neurite density index (NDI) in the spinal cord white matter in relapsing-remitting MS patients (scatterplot).

Data are shown with confidence intervals computed the standard error of forecast due to the non-parametric variable. In relapsing-remitting MS patients the EDSS score was correlated a lower neurite density (NDI) in the spinal cord white matter $\left(\mathrm{r}_{\mathrm{s}}=-0.46 \mathrm{p}=0.015\right)$.

Abbreviations: EDSS: Expanded Disability Status Scale; NDI: neurite density index; WM: white matter. 


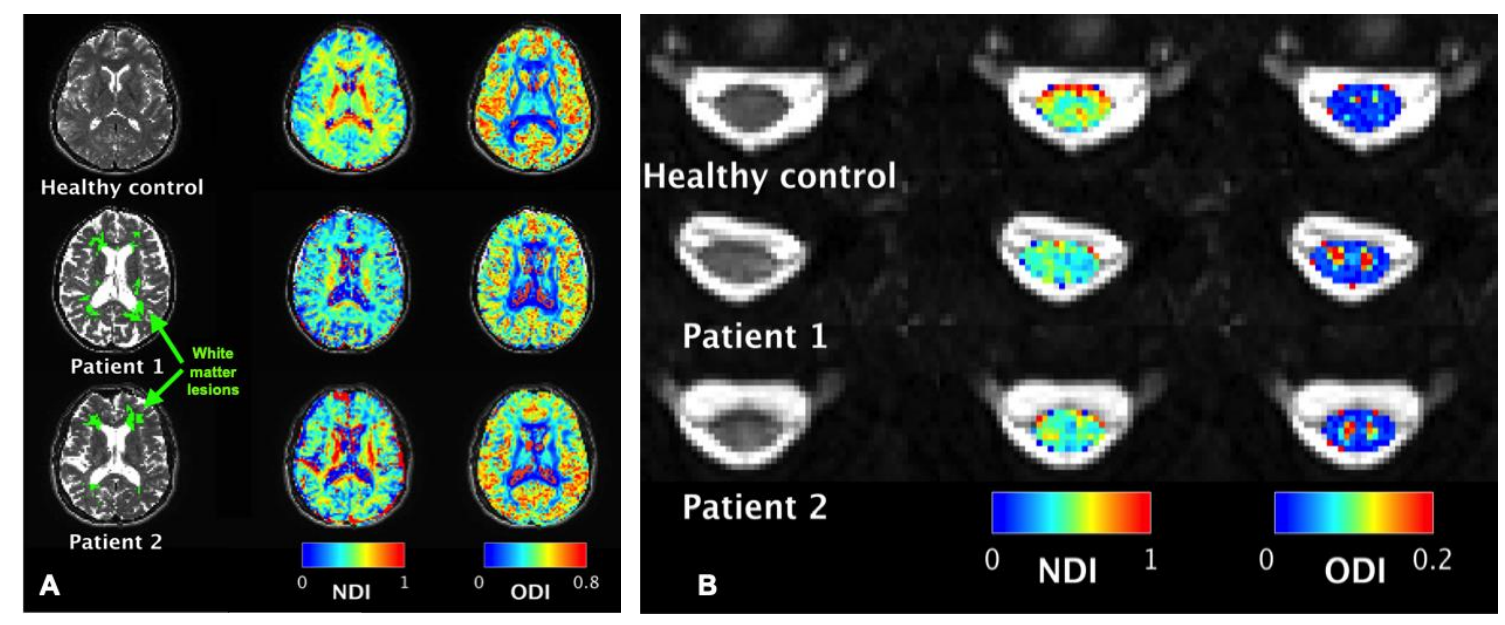




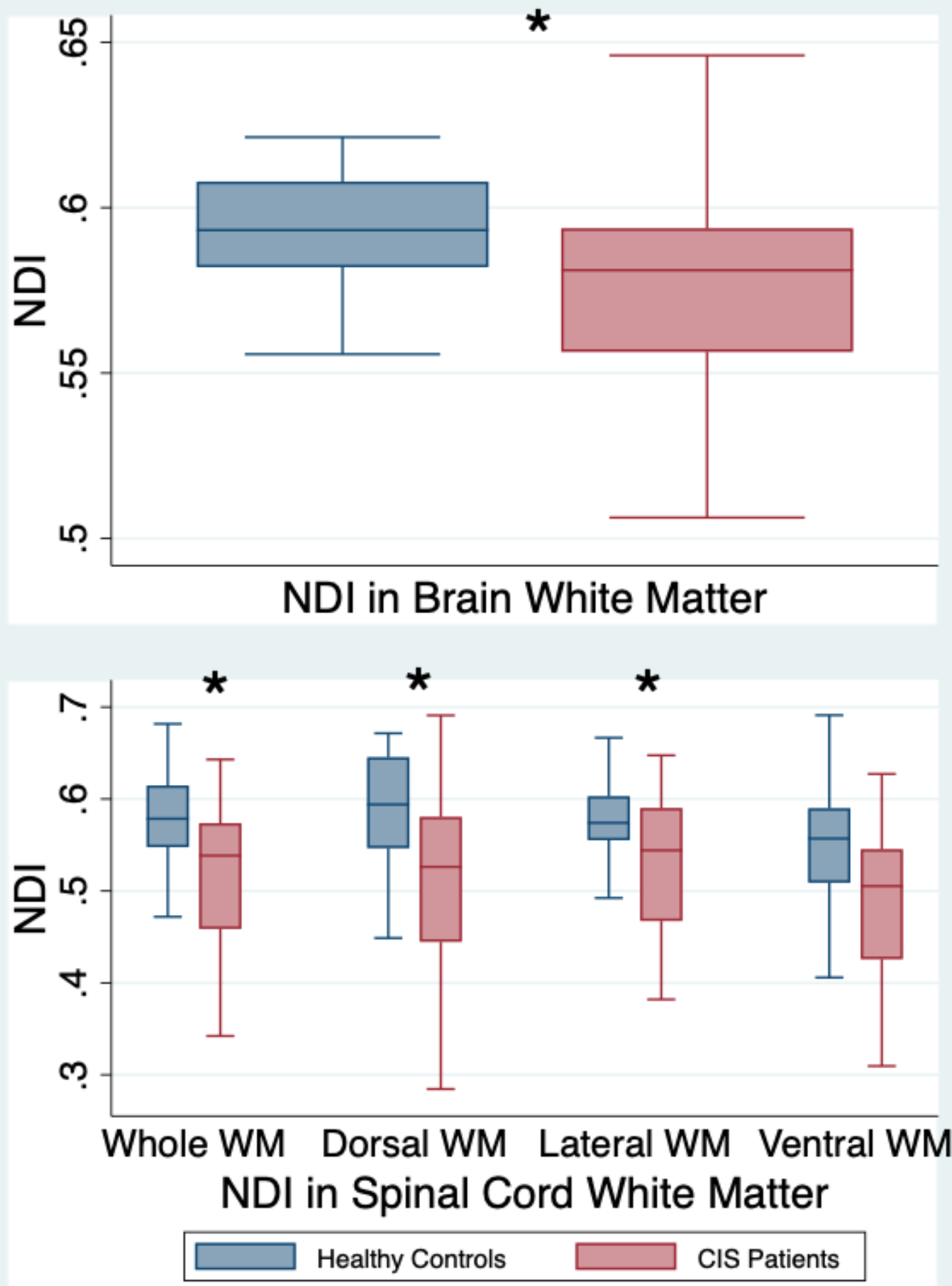


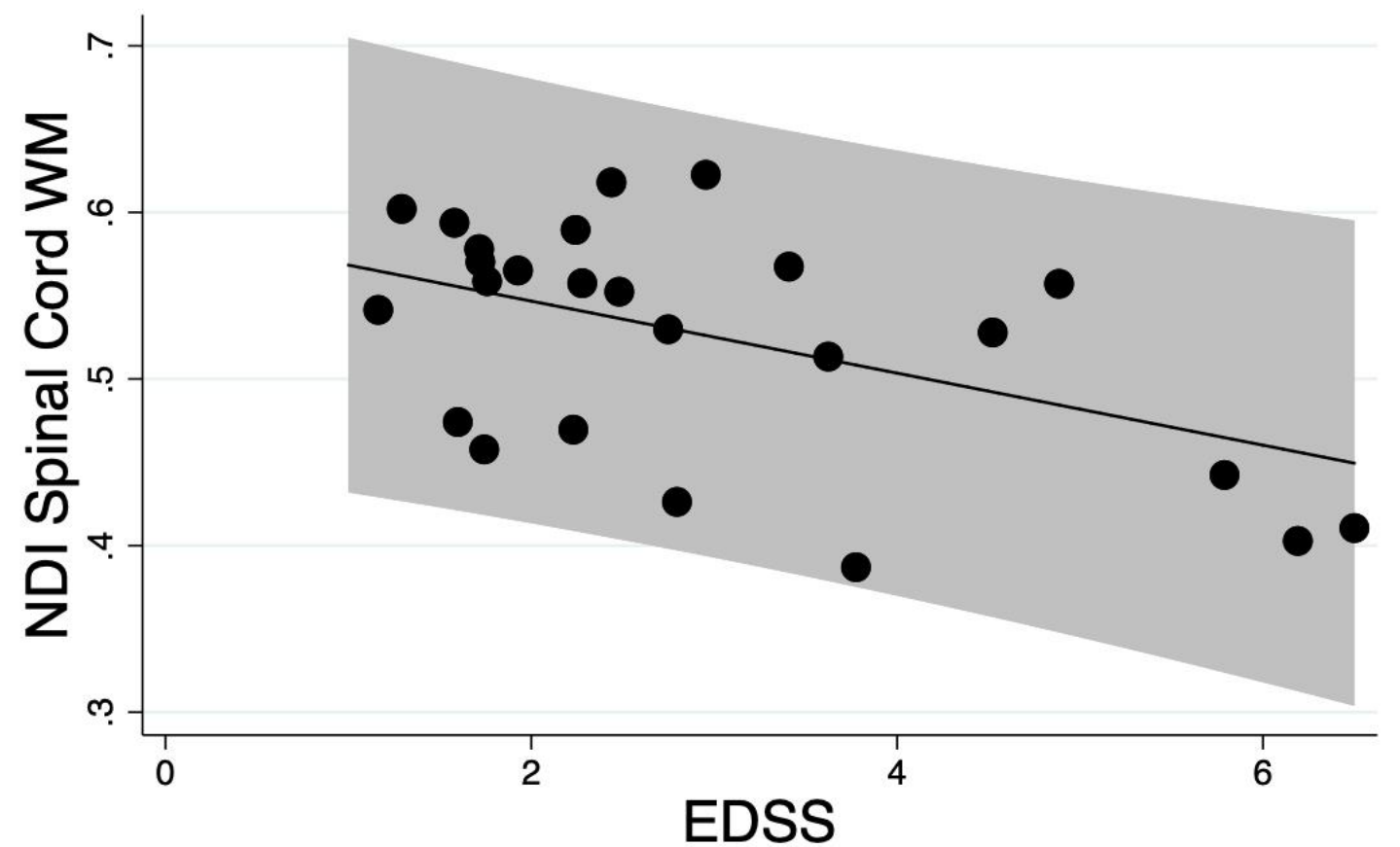

$95 \% \mathrm{Cl} \longrightarrow$ Fitted values 